\title{
Comparison of Print Quality on Papers From InVASIVe Alien Plants SPECIES
}

\author{
SELIČ, P.; MAVRIĆ, Z. \& MOŽINA, K.
}

Abstract: In nature there are more and more problems caused by invasive alien plant species. They not only threaten and displace native plants, but also cause economic damage. The aim of this study was to investigate and determine the percentage of weeking of prints on papers made from invasive alien plant species, i.e. Japanese knotweed and goldenrod. The same pattern was printed on both invasive alien plant species papers, Japanese knotweed and goldenrod, using two different printing techniques, i.e. inkjet and electrophotographic. We measured the percentage of the appearance of the week on both printing techniques. The highest percentage deviation of $9.42 \%$ was found on paper made of cellulose fibres obtained from goldenrod using the inkjet printing process, while the deviation from the ideal print using the electrophotographic printing process on the same paper was $2.02 \%$. From the research results we can conclude that the electrophotographic printing technique is more suitable for printing on papers made from invasive alien plant species.

Key words: Japanese knotweed, goldenrod, inkjet print, electrophotographic, weeking
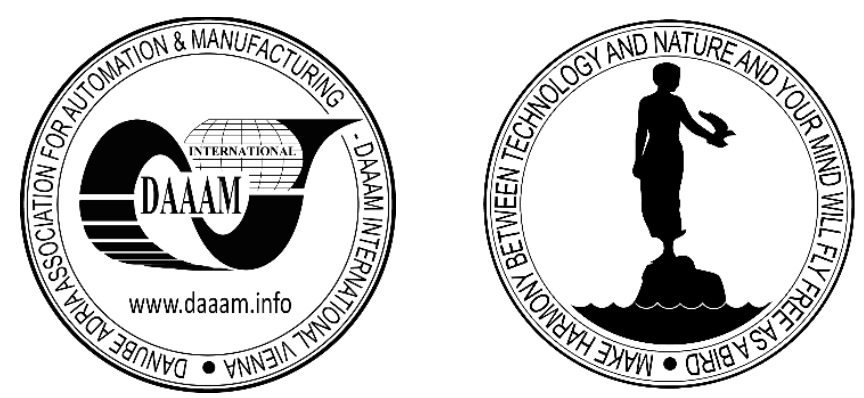

Authors' data: Selič, P[atricija]; Mavrić, Z[ala] \& assist. prof. dr. Možina, K[lemen], Faculty of Natural Sciences and Engineering, University of Ljubljana, Snežniška ulica 5, 1000, Ljubljana, Slovenia, klemen.mozina@ntf.uni-lj.si

This Publication has to be referred as: Selic, P[atricija]; Mavric, Z[ala] \& Mozina, K[lemen] (2020). Comparison of Print Quality on Papers from Invasive Alien Plants Species, Chapter 04 in DAAAM International Scientific Book 2020, pp.049-060, B. Katalinic (Ed.), Published by DAAAM International, ISBN 978-3-902734-27-3, ISSN 1726-9687, Vienna, Austria

DOI: $10.2507 /$ daaam.scibook.2020.04 
Selic, P.; Mavric, Z. \& Mozina, K.: Comparison of Print Quality on Papers from Inv..

\section{Introduction}

Invasive alien species are a growing problem for the environment and human health. They can lead to loss of biodiversity and changes in ecosystem functioning (Sterzyńska et al., 2017). Their influence stems from their ability to maintain a diverse ecosystem with a single plant species.

They directly threaten and influence the structure of habitats, abiotic properties of the soil, changes in the chemical structure of the soil, geomorphological processes, diversity and functioning of the animal community (Lavallée et al., 2019; Možina et al., 2020). Invasive plants contribute to the decomposition of flora, as their invasiveness often reduces the biodiversity of natural ecosystems (Cai et al., 2020). However, they also have major economic impacts, as the economic costs of controlling and maintaining established non-native plants are very high (Možina et al., 2020).

Due to the global and local impacts, there is a growing need for action, early detection, awareness raising and rapid response, which is crucial to prevent further spread of these species (Lavallée et al., 2019; Možina et al., 2020). The question is how invasive alien plants can be effectively eliminated so that they are not only removed from the wild but can also be put to beneficial use. An area is emerging where invasive alien plants and their parts can be processed into, for example, pigments and wooden parts for a range of useful products such as furniture, decorations, etc. Among other things, cellulose fibers can also be used in paper production, i.e. manually and mechanically.

The Japanese knotweed (Fallopia japonica) is an invasive perennial shrub-like growth native to Japan. It is one of the 100 most invasive plants in the world. Its planting is prohibited in the EU. The Japanese knotweed can be recognized by its bushy, 3 to 4-meter-high form. It has a curved, jointed, hollow stem that resembles bamboo. In winter, the above-ground parts die off and the underground rhizomes, which can reach several meters, die off. It has a very branched underground root system, which can be up to $20 \mathrm{~m}$ long and reach 2 to $3 \mathrm{~m}$, in some cases even $5 \mathrm{~m}$ deep. The leaves have entire margins and are broadly ovate, 5 to $20 \mathrm{~cm}$ long, up to $10 \mathrm{~cm}$ wide.

The base is truncated. It flowers from the end of July to October and has tiny whitish flowers grouped in pubescent inflorescences. It has five petals, the outer three of which touch the broad edges and surround the developing fruit, a triangular nut that turns black at maturity. The flowers are unisexual, in Europe only female, as all plants are descendants of the same "mother", but they can be pollinated by pollen of the Sakhalin knotweed, and hybrids are formed, e.g. the Czech knotweed (Eler, 2018; Lešnik, 2017; Berden, 2019). It effectively adapts its growth to environmental conditions and is therefore one of the most invasive alien species in the world, but it can also have positive effects and help certain ecosystems (Možina et al., 2020). 
The plant can be used as a source of food, dyes or cellulose fibres that can be used in paper production (Eler, 2018). The giant goldenrod (Solidago gigantea) comes from North America and grows up to $2 \mathrm{~m}$ high. It flowers from July to October and has yellow flowers grouped into tiny pieces arranged in branched inflorescences. The fruit is a 0.9 to $1.2 \mathrm{~mm}$ large nut with a long tuft of hairs about $2.5 \mathrm{~mm}$ in size. The seeds are ripe from September. Giant goldenrod has a smooth stem and the ability to reproduce vegetatively by rhizomes from which new shoots grow, forming very dense stands.

It is also an insect flower. After fertilization, many fruits develop from tufts of hair carried by the wind (Eler, 2018; Lešnik, 2017; Berden, 2019). Habitats for goldenrod can be found in various parts of the soil, especially in the lowlands, on low hills, dry meadows, in forests, along roads and railway lines, in abandoned fields, along watercourses, bogs and clearings.

The invasion of goldenrod brings about a strong change in the physical-chemical properties of the soil and causes significant changes in the above-ground communities of arthropods at different trophic levels (Sterzyńska et al., 2017). Goldenrod is very popular among beekeepers because of its rich autumn grazing and in traditional medicine for wound healing and urinary tract inflammation.

Giant goldenrod contains a larger number of flavonoids. It also contains triterpene saponins and phenolic acids, which contribute to its healing properties. Like the Japanese knotweed, goldenrod can also be used as a food source, dye or cellulose fiber, which can be used in paper production (Eler, 2018; Lešnik, 2017; Berden, 2019).

Some solutions, such as jewellery packaging and packaging for invasive wood microscopy samples, have already been developed as part of the Applause project. However, there was a problem with printing on this type of material. This is still a rather unexplored area, and due to the nature of paper, it is difficult to predict how paper will behave during the printing process (Berden, 2019).

The purpose of this study was to determine the degree of distortion, i.e. the degree of print cloudiness on paper made from invasive plants, namely paper made from Japanese knotweed, goldenrod and "ordinary" office paper. On all papers the same print template (PT) was printed with two different printers (inkjet and electrophotographic), which were used for more detailed observations and investigations. The aim was to find out whether papers from invasive alien plant species could be used for daily printing needs. The first main question was which printing technique produces less distortion on invasive alien plants and the second was the percentage difference between samples of PT on "ordinary" office paper and papers made from invasive alien plants and how the basic physical properties determine the quality of the prints. 
Selic, P.; Mavric, Z. \& Mozina, K.: Comparison of Print Quality on Papers from Inv..

\section{Experimental}

In the experimental part we investigated the basic (thickness, grammage, density, specific volume and moisture content), surface (roughness, porosity and water absorption capacity), printing (print penetration) and structural properties (capillary rise) of papers and the image analysis of overprints.

\subsection{Materials and Methods}

\subsubsection{Materials}

Three paper samples were used in the study:

1. Japanese knotweed consists of $40 \%$ cellulose fibres of Japanese knotweed, 35\% eucalyptus and 25\% unbleached thermomechanical spruce fibre pulp (TMP). Besides the cellulose fibres, the paper also contains retention agent $(0.03 \%)$, alkyl ketene dimer $(\mathrm{AKD}, 2.5 \%)$, cationic agent $(0.75 \%)$, starch $(0.03 \%)$ and $\mathrm{CaCO} 3$ fillers $(6.5 \%)$.

2. Goldenrod, consisting of $45 \%$ goldenrod cellulose fibres, $30 \%$ eucalyptus and $25 \%$ unbleached thermal mechanical pulp (TMP). The remaining accessories correspond to the range specified in the Japanese knotting paper sample.

3. "Ordinary" office paper for which precise compositional data are not available due to the manufacturer's trade secret and which consists of wood cellulose fibers from spruce, pine, eucalyptus and birch, with a conventional $\mathrm{CaCO} 3$ filler and other "standard" additives.

\subsubsection{Basic properties}

In order to determine the basic characteristics of the machine-made paper under investigation, i.e. Japanese knotweed, goldenrod and "ordinary" office paper on which the prints were made, we determined the thickness (ISO 534, 2005), grammage (ISO 536, 2019), the density (ISO 534, 2005), the specific volume (ISO 534, 2005) and the moisture content (ISO 287, 2017) (Table 1).

The papers included in the investigation were machine-made. Paper from invasive alien plants, i.e. Japanese knotweed and goldenrod, on the Andritz paper machine (Andritz AG, Graz, Austria, located at the Institute for Pulp and Paper, Ljubljana, Slovenia) and "ordinary" office paper on paper machine 4 (PM4), Voith Paper Krieger, Mönchengladbach, Germany, located at Radeče papir Nova, Radeče, Slovenia).

\begin{tabular}{|c|c|c|c|c|c|}
\hline & $\begin{array}{c}\mathrm{d} \\
{[\mathrm{mm}]}\end{array}$ & $\begin{array}{c}\mathrm{G} \\
{\left[\mathrm{g} / \mathrm{m}^{2}\right]}\end{array}$ & $\rho\left[\mathrm{kg} / \mathrm{m}^{3}\right]$ & $\begin{array}{c}v \\
{\left[\mathrm{~m}^{3} / \mathrm{kg}\right]}\end{array}$ & $\begin{array}{c}\mathrm{V} \\
{[\%]}\end{array}$ \\
\hline $\begin{array}{l}\text { Japanese } \\
\text { knotweed }\end{array}$ & $\begin{array}{r}0.160 \pm \\
0.002\end{array}$ & $96.2 \pm 4.2$ & $\begin{array}{r}613 \pm \\
20\end{array}$ & $\begin{array}{r}1.63 \pm \\
0.05\end{array}$ & $\begin{array}{r}5.0 \pm \\
0.1\end{array}$ \\
\hline goldenrod & $\begin{array}{r}0.170 \pm \\
0.002 \\
\end{array}$ & $88.6 \pm 1.1$ & $\begin{array}{r}517 \pm \\
9\end{array}$ & $\begin{array}{r}1.93 \pm \\
0.03 \\
\end{array}$ & $\begin{array}{r}4.9 \pm \\
1.6 \\
\end{array}$ \\
\hline office paper & $\begin{array}{r}0.130 \pm \\
0.001\end{array}$ & $100.0 \pm 0.8$ & $799 \pm$ & $\begin{array}{r}1.25 \pm \\
0.01 \\
\end{array}$ & $\begin{array}{r}3.5 \pm \\
1.6\end{array}$ \\
\hline
\end{tabular}

Tab. 1. Basic properties 


\subsubsection{Surface and printing properties}

The surface properties of the paper were defined by a series of measurements that provide more detailed information about how the substrate surface influences the print quality, since the quality of the print depends largely on the surface quality of the paper on which it is printed.

Roughness defines the surface uniformity of the paper, which has a direct effect on the overflow, while the degree of porosity influences the serration of the imprint (ISO 8791-2, 2013).

The degree of hydrophilicity was measured with the Cobb method of surface water absorption according to (ISO 535, 2014). The surface treatment of the paper was also examined with the printing properties, i.e. the printing penetration (PP) (IGT AIC2-5T2000 Global Standard Tester 1/1-W), which is a measure of the ability to absorb printing ink and depends on the absorbency, roughness and porosity of the printing material (Table 2).

\begin{tabular}{|l|r|r|r|r|r|r|r|}
\cline { 2 - 8 } \multicolumn{1}{c|}{} & \multicolumn{1}{c|}{$\mathrm{H}_{\mathrm{B}}[\mathrm{ml} / \mathrm{min}]$} & \multicolumn{2}{c|}{$\mathrm{P}_{\mathrm{B}}[\mathrm{ml} / \mathrm{min}]$} & \multicolumn{2}{c|}{$\mathrm{C}_{60}\left[\mathrm{~g} / \mathrm{m}^{2}\right]$} & $\mathrm{PP}[/]$ \\
\cline { 2 - 9 } \multicolumn{1}{c|}{} & \multicolumn{1}{c|}{$\mathrm{A}$} & \multicolumn{1}{c|}{$\mathrm{B}$} & $\mathrm{A}$ & $\mathrm{B}$ & $\mathrm{A}$ & $\mathrm{B}$ & $\mathrm{MD}$ \\
\hline Japanese & $1336 \pm$ & $1580 \pm$ & $324 \pm$ & $328 \pm$ & $19.8 \pm$ & $21.4 \pm$ & $30 \pm$ \\
knotweed & 155 & 84 & 18 & 23 & 1.6 & 2.0 & 3.84 \\
\hline \multirow{2}{*}{ goldenrod } & $1250 \pm$ & $1570 \pm$ & $1790 \pm$ & $1730 \pm$ & $18.7 \pm$ & $20.6 \pm$ & $39 \pm$ \\
& 146 & 115 & 103 & 76 & 3.4 & 3.7 & 0.84 \\
\hline \multirow{2}{*}{ office paper } & $216 \pm$ & $164 \pm$ & $500 \pm$ & $506 \pm$ & $20.0 \pm$ & $19.8 \pm$ & $25 \pm$ \\
& 65 & 18 & 35 & 26 & 1.3 & 1.4 & 0.56 \\
\hline
\end{tabular}

Tab. 2. Surface and printing properties

\subsubsection{Structural properties}

Capillary rise - Klemm method (Table 3 ) is a property of paper to absorb water in a vertical direction which, according to the principle of capillarity, rises above the water level in which the paper sample is immersed and is closely correlated with the overflow rate of the imprint produced (ISO 8787, 1986).

\begin{tabular}{|l|r|r|}
\cline { 2 - 3 } \multicolumn{1}{c|}{} & \multicolumn{2}{c|}{$1[\mathrm{~mm}]$} \\
\cline { 2 - 3 } \multicolumn{1}{c|}{} & MD & CD \\
\hline Japanese knotweed & $14.4 \pm 1.1$ & $16.2 \pm 3.9$ \\
\hline Office paper & $14.8 \pm 3.0$ & $15.0 \pm 1.7$ \\
\hline
\end{tabular}

Tab. 3. Capillary rise - Klemm method 
Selic, P.; Mavric, Z. \& Mozina, K.: Comparison of Print Quality on Papers from Inv..

\subsubsection{Printer properties}

The test form was printed on a inkjet printer Brother DCP-T510W and on an electrophotographic, i.e. laser printer, Canon ImageRunner C5045i. The colour prints are shown in Table 4. The printing form were printed with the original pigment-based ink. As can be seen from the test form (Table 4), two sans serif fonts were used, i.e. Arial and Times New Roman in different sizes from 2.2 to 73.5 pt. For the quantitative evaluation of the derived prints the number eight was used, i.e. in Arial an in the size $7.4 \mathrm{pt}$.

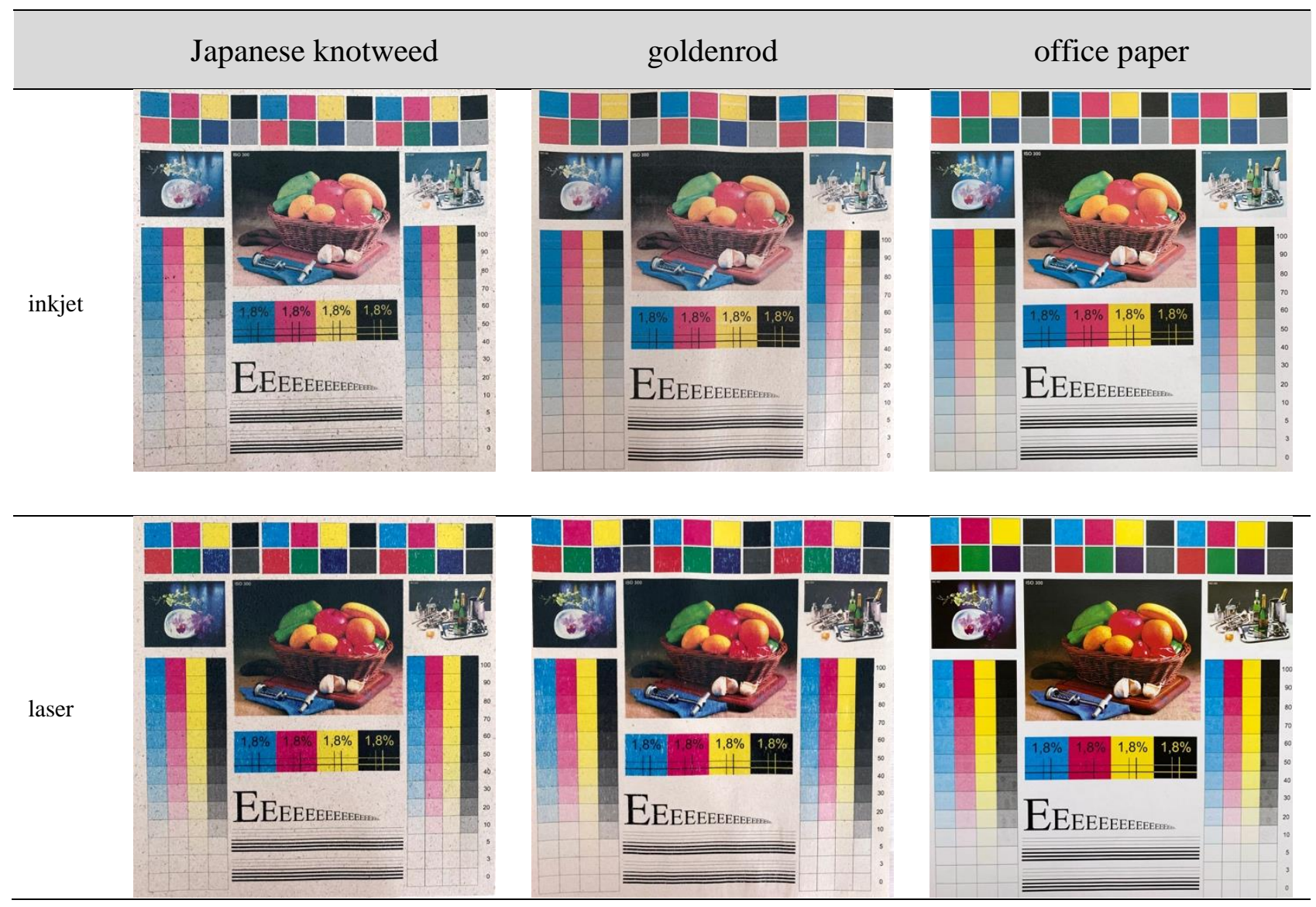

Tab. 4. Prints of test form

\subsubsection{Capture of images prints}

The images of the prints were taken with a Leica S9I Stereozoom microscope, at $2 \mathrm{x}$ magnification and in full colour space. The size of the captured image was 0.8 MP $(1024 \times 768 \mathrm{px})$; ratio $4: 3$. The brightness was adjusted to $70 \%$. The image saturation was 147 and the gamma value was 0.45 .

\subsubsection{Black/white ratio}

The ratio between black and white was determined using three samples for each paper type and the selected printer. The images were first Adobe Photoshop processed in where they were converted to black and white (threshold settings: 228). 
They were then corrected in Adobe Photoshop, where the resulting printing errors and the photos themselves were filled in on the images where appropriate. Only images of a certain section were compared, but always in the same size of $47.75 \times$ $70.19 \mathrm{~mm}$. For the final, edited images, the ImageJ program used a script to calculate the ratio between white and black dots, i.e. the Calculate Black /white ratio. Starting from the basic printing form, i.e. the ideal computer form, we calculated the percentage of deviation/expiration, the weekly delay, from the selected printed graphic character, i.e. the number 8 .

\section{Results and discussion}

\subsection{Basic paper properties}

The grammage of the papers investigated is suitable for printing leaflets, brochures and letterheads, as the average was $96.2 \mathrm{~g} / \mathrm{m}^{2}$ for Japanese knotweed paper and $88.6 \mathrm{~g} / \mathrm{m}^{2}$ for goldenrod paper. Flyers, brochures and letterheads are usually printed using the inkjet printing process rather than electrophotographic printing technique. The moisture content of paper made from invasive foreign plants was 5.0\%, which is quite a high value as it has a noticeable effect on the print quality, such as waviness, flatness and the ability to transfer ink.

\subsection{Surface and printing properties of papers}

\subsubsection{Roughness and porosity}

The roughness of both paper types from invasive alien plants is comparable, i.e. $1336-1580 \mathrm{ml} / \mathrm{min}$, while office paper has a roughness that is $8 \times$ times lower roughness $(190 \mathrm{ml} / \mathrm{min})$.

The porosity measurements show greater differences between the papers examined, with Japanese knotweed paper showing the lowest degree of porosity or the most closed paper surface $(325 \mathrm{ml} / \mathrm{min})$, followed by office paper $(503 \mathrm{ml} / \mathrm{min})$ and goldenrod, which has a $5.5 \times$ times higher degree of porosity $(1760 \mathrm{ml} / \mathrm{min})$ than Japanese knotweed (Table 2). It is expected that papers with a higher degree of porosity will have a greater tendency to warp, especially when using wet printing techniques such as drop or inkjet printing.

\subsubsection{Surface water absorption and printing penetration}

The surface water absorption capacity according to the Cobb method after 60 seconds of water impression on the surface of the papers examined was about $20 \mathrm{~g} / \mathrm{m} 2$ for all samples examined (Table 2), which indicates that the paper is fully sized and complies with the recommendations for such special papers, i.e. leaflets, brochures and letterheads. 
Selic, P.; Mavric, Z. \& Mozina, K.: Comparison of Print Quality on Papers from Inv..

The full degree of adhesion is reflected in the degree of hydrophobicity of the paper, which was confirmed by measurements of print penetration. In all paper samples tested, print penetration was between 25 and 30 (Table 2), which directly indicates the low ability of the aqueous substrate dibutylphthalate (DBF) to absorb the water. The ink remains on the surface of the printing medium, resulting in more colour saturated and high-contrast prints.

\subsection{Visual evaluation of test forms}

The visual assessment results in better quality of prints produced by dry printing process, i.e. electrophotography, which is expected and predicted due to the lack of liquid medium, water, and therefore offers a lower rate of both interlocking and weeking.

In the case of inkjet printing, i.e. in the presence of an aqueous medium, the structural properties have a great influence on the quality of the prints made, although there are no significant differences between the papers examined, i.e. colour saturation, jagged edges and curvature (Table 4). The same tendency in print quality can be observed in the electrophotographic printing technique.

\subsection{Image analysis of prints}

Table 5 shows the images of the prints produced on the test papers examined using two printing techniques. The images taken with a microscope clearly show the deviation from the computer-generated graphic image of the number 8, with a more pronounced weeking is observed on the samples printed with liquid toner, i.e. using the droplet printing process.

Japanese knotweed goldenrod $\quad$ office paper $\begin{gathered}\text { ideal/ } \\ \text { computer shape }\end{gathered}$


laser
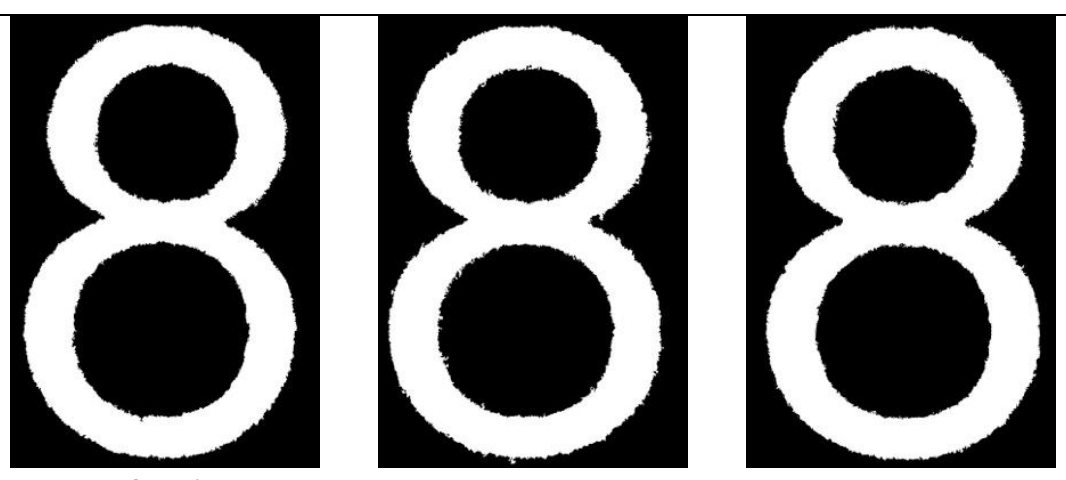

Tab. 5. Images of prints

As can be seen from the capillary absorbency according to the Klemm method (Table 3), in which the values between the samples of the examined papers from invasive alien plants are quite similar, i.e. in MD, the vertical absorption in both paper samples is 14.4 (Japanese knotweed) and $14.8 \mathrm{~mm}$ (goldenrod), while the Klemm value for office paper is $20.2 \mathrm{~mm}$, i.e. a $38.36 \%$ higher capillary absorbency, which, however, does not lead to a stronger week formation due to capillarity.

On the contrary, the values are quite comparable and range between 8 and 10\% (Table 6), with the lowest value being measured for office paper with the lowest roughness (Table 2).

\begin{tabular}{|l|r|c|}
\cline { 2 - 3 } \multicolumn{1}{c|}{} & \multicolumn{2}{c|}{ PF [\%] } \\
\cline { 2 - 3 } \multicolumn{1}{c|}{} & inkjet & laser \\
\hline Japanese knotweed & 8.97 & 1.39 \\
\hline goldenrod & 9.86 & 2.66 \\
\hline office paper & 8.08 & 1.28 \\
\hline
\end{tabular}

Tab. 6. Percentage of deviation of the white area from the ideal printing form

When comparing the two types of paper studied, made from invasive alien plants, the highest degree of distortion of the print on paper made from giant goldenrod was $9.86 \%$ in inkjet printing and $2.66 \%$ in electrophotographic printing technique (Table 6).

The higher degree of weeking, in the paper samples made from goldenrod is due to the significantly higher degree of porosity compared to the paper samples made from the Japanese knotweed plant, where the porosity of the paper made from goldenrod is $5.5 \times$ higher, i.e. $1790 \mathrm{ml} / \mathrm{min}$ (Table 2).

From the measurement results we can conclude that electrophotographic printing is more suitable for printing on paper made from invasive, alien species and on "ordinary" office paper because of less distortion and weeking.

The electrophotographic printing technique would allow larger amounts of text and smaller font sizes to be printed on paper made from invasive alien species and 
Selic, P.; Mavric, Z. \& Mozina, K.: Comparison of Print Quality on Papers from Inv..

would still be legible, unlike the inkjet printing technique, which would cause distortion of the print and therefore poor legibility.

Electrophotographic printing techniques could also be used to print decorative, thinner lines and, last but not least, photographs and diagrams, as the prints proved to be sufficiently contrasty (Table 5).

\section{Conclusions}

Invasive alien plants have a broad impact on ecosystems due to their rapid spread and adaptation to different ecosystems. Ecologists and researchers are trying to find ways to prevent their further spread. Due to their ability to form fibre networks, invasive alien plants can also be used in paper production. The aim of the study was to determine and compare the degree of jagged and weeking of imprints on paper made from cellulose fibres of non-native invasive plants, i.e. Japanese knotweed and goldenrod, and on "ordinary" office paper.

The research results lead to the following conclusions:

1. major deviations from the ideal computer representation of the number 8 exist in the case of a print form printed in a wet printing process, i.e. with an inkjet printer;

2. the maximum deviations from the computer printout number 8 exist when printed on paper made of cellulose fibres of the invasive alien plant, i.e. goldenrod, using both types of printer, wet and dry;

3. for printing on paper using a raw material derived from invasive alien plant species, it can be concluded, on the basis of the measurements carried out in the research, that electrophotographic printing technology is more suitable among the two types of paper presented and Japanese knotweed paper is more suitable among two types of paper studied which were produced from invasive alien plant species;

4. percentage of weeking and jagging on "ordinary" office paper is slightly lower for both printing techniques than on paper made from invasive alien plants.

On the basis of the results we can conclude that laser printing technology is more suitable for prints on paper from invasive alien plants, so that it can be used for everyday printing. For further research, we should test different types of graphic projects such as brochures, leaflets and other graphic designs on invasive alien plants and see how people would adapt to them.

We could also test the print quality on other reproduced materials, as well as other features besides weeking. This could be tested by measuring colour deviations, i.e. coloured images with a spectrophotometer. In this way we could determine the best printing and preparation properties for printing on invasive paper. 
By printing documents on invasive alien plant paper every day, we can help our ecosystems by preventing the further spread of invasive alien plant species.

\section{Acknowledgements}

This research is a part of the project UIA02-228 APPLAUSE - Alien Plant Species, from harmful to useful with citizens' led activities. Project is co-financed by the EU Regional Development Fund.

\section{References}

Berden, S. (2019). APPLAUSE - From harmful to useful non-native plants with active involvement of the citizen: recognize, reuse or hand over. City of Ljubljana, Department of Environmental Protection. ISBN 978-961-6449-80-9, Ljubljana, Slovenia.

Cai, H.; Lu, H.; Tian, Y.; Liu, Z.; Huang, Y. \& Jian S. (2020). Effects of invasive plants on the health of forest ecosystems on small tropical coral islands, Ecological Indicators, Vol. 117, ISSN 1470-160X, China.

Calvert, P. (2001) Inkjet Printing for Materials and Devices. Chemistry of Materials, Vol. 13, No. 10, 3059-3809, ISSN 3299-3305.

Eler, K. (2018). Invasive plants and agriculture, University of Ljubljana, Biotechnical faculty, ISBN 978-961-6761-57-4, Ljubljana, Slovenia.

IGT AIC2-5T2000 Global Standard Tester 1/1-W - IGT Testing Systems; 2006, 2p.

ISO 534: Paper and board - Determination of thickness, density and specific volume; 2005, 13 p.

ISO 536: Paper and board - Determination of grammage; 2019, 8 p.

ISO 287: Paper and board - Determination of moisture content of a lot - Oven-drying method; 2017, $10 \mathrm{p}$.

ISO 8791-2: Paper and board - Determination of roughness/smoothness (air leak methods) - Part 2: Bendtsen method; 2013, 16 p.

ISO 535: Paper and board - Determination of water absorptiveness - Cobb method; 2014, 8 p.

ISO 8787: Paper and board - Determination of capillary rise - Klemm method; 1986, $2 \mathrm{p}$.

Lavallée, F.; Smadi, C.; Alvarez, I.; Reineking, B.; Martin, F.M.; Dommanget, F. \& Martin, S. (2019). A stochastic individual-based model for the growth of a stand of Japanese knotweed including mowing as a management technique, Ecological Modelling, Vol. 413, ISSN 0304-3800.

Lešnik, M. (2017). Controlling the occurrence of invasive (neophytes) and conservation of biodiversity in water protected areas, University press of Maribor, ISBN 978-961-286-122-3, Maribor, Slovenia. 
Selic, P.; Mavric, Z. \& Mozina, K.: Comparison of Print Quality on Papers from Inv..

Možina, K.; Bračko, S.; Kovačević, D.; Blaznik, B. \& Možina, K. (2020). Japanese Knotweed Paper as Printing Substrate, BioResources, Vol. 15, No. 2, 3999-4015, ISSN 2000-0669

Sterzyńska, M.; Shrubovych, J. \& Nicia, P. (2017). Impact of plant invasion (Solidago gigantea L.) on soil mesofauna in a riparian wet meadows, Pedobiologia - Journal of Soil Ecology, Vol. 64, 1-7, ISSN 0031-4056. 